\title{
ACTRIS AND ITS AEROSOL REMOTE SENSING COMPONENT
}

\author{
Ulla Wandinger*, ${ }^{*}$, Doina Nicolae ${ }^{2}$, Gelsomina Pappalardo ${ }^{3}$, Lucia Mona ${ }^{3}$, Adolfo Comerón ${ }^{4}$ \\ ${ }^{1}$ Leibniz Institute for Tropospheric Research, Leipzig, Germany \\ ${ }^{2}$ National Institute of $R \& D$ for Optoelectronics, Magurele, Romania \\ ${ }^{3}$ Istituto di Metodologie per l'Analisi Ambientale, Tito Scalo, Potenza, Italy \\ ${ }^{4}$ Universitat Politècnica de Catalunya, Barcelona, Spain \\ *Email: ulla@,tropos.de
}

\begin{abstract}
The Aerosol, Clouds and Trace Gases Research Infrastructure ACTRIS is currently being developed with support from more than 20 countries and more than 100 research-performing organizations in Europe. The pan-European distributed research infrastructure shall provide data and services related to short-lived atmospheric constituents to facilitate high-quality Earth system research in the long term (over at least 20 years). While some of the activities are already in place, ACTRIS functionality will be further ramped up until full operation in 2025 . The observation of aerosol, clouds and reactive trace gases with in-situ and remote-sensing techniques in ACTRIS is supported by six Topical Centres, which are responsible for common standards and quality assurance. Free and open virtual access to ACTRIS data is provided by the Data Centre. International users will also have physical access to ACTRIS observatories, atmospheric simulation chambers and mobile platforms as well as remote or physical access to calibration services, digital services and training. Access provision is organized through a singleentry point by the Head Office. In this contribution, the general principles and structure of ACTRIS are introduced, and the observational component related to aerosol remote sensing, which builds on the heritage of the European Aerosol Research Lidar Network (EARLINET) and the European part of the Aerosol Robotic Network (AERONET-Europe), is explained in more detail.
\end{abstract}

\section{ACTRIS OBJECTIVE}

ACTRIS intends to provide data and services related to the 4D-variability and the physical and chemical properties of short-lived atmospheric constituents and the processes leading to their formation, transformation and removal. ACTRIS data and services shall facilitate and enhance research in atmospheric science, with particular focus on climate change and air quality, and support the worldwide user community working on models, satellite retrievals and analysis and forecast systems. An overview on the current status of ACTRIS can be found in the ACTRIS Stakeholder Handbook 2018 [1].

\section{ACTRIS STRUCTURE}

ACTRIS is organized around Central Facilities and National Facilities as shown in Figure 1.

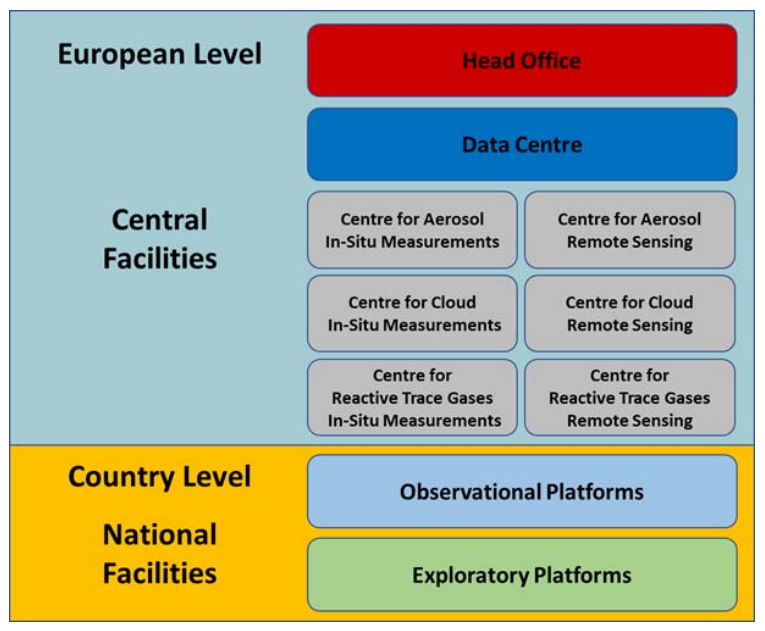

Figure 1: ACTRIS structure consisting of eight Central Facilities operated at European level and National Facilities comprising Observational and Exploratory Platforms operated at the national level.

ACTRIS Central Facilities represent the key operative entities of the research infrastructure and have a fundamental role as they provide data and services to users according to the ACTRIS policies $[2,3]$ as well as operation support to the National Facilities. ACTRIS Central Facilities are the Head Office, the Data Centre, and six Topical 
Centres. The Central Facilities will be established at the European level and will be part of the governance and decision-making structure of ACTRIS. Each Central Facility has several operational Units, which are typically located at national research-performing organizations with respective expertise in different countries.

ACTRIS Topical Centres are organized around the main scientific themes of ACTRIS: aerosol, clouds and reactive trace gases, each with a particular focus on either remote sensing or in-situ measurement techniques (see Figure 1). The key services and operation support provided by the Topical Centres will be

a) To develop procedures and tools for quality assurance and quality control (QA/QC) of ACTRIS measurements and data,

b) To perform knowledge transfer and training to ACTRIS operators and users,

c) To foster the implementation of validated new techniques in ACTRIS,

d) To stimulate new techniques and methodologies and to sustain a high level of performance,

e) To contribute to expert collaboration networks.

ACTRIS National Facilities consist of Observational and Exploratory Platforms, which are developed, managed and operated by researchperforming organizations at the national level. Observational Platforms are fixed ground-based stations that deliver long-term data based on a regular measurement schedule and common operation standards. They are widely distributed over Europe and also located at selected sites outside of Europe. Observational Platforms must contribute to at least one of the six observational components, i.e., remote sensing or in-situ measurement of aerosol, clouds and reactive trace gases, following the principles and technical requirements of ACTRIS [4].

Exploratory Platforms are atmospheric simulation chambers, laboratory platforms and mobile platforms that perform dedicated experiments and contribute data on atmospheric compounds, processes, events or regions by following common ACTRIS standards [5]. The major task of the National Facilities is the acquisition and delivery of quality-controlled data. National
Facilities, in particular the exploratory platforms and large observatories, shall also provide physical access to users, which will be centrally managed by the Service and Access Management Unit within the ACTRIS Head Office.

\section{LEGAL IMPLEMENTATION}

ACTRIS will be implemented as a European Research Infrastructure Consortium (ERIC), a legal entity based on an agreement of EU Member States and associated countries. The task of ACTRIS ERIC is to establish and operate the distributed research infrastructure, including liaisons and establishment of long-term, sustainable and formal agreements with ACTRIS Central Facilities and management of the service provision for a wide user community. ACTRIS ERIC will provide the coordination, integration, development, monitoring and governance of ACTRIS and is in charge of the strategic and financial development and eventual long-term operation and sustainability of ACTRIS. The tasks and activities of ACTRIS ERIC are defined in the ACTRIS ERIC Statutes [6].

\section{TECHNICAL REQUIREMENTS FOR AEROSOL REMOTE SENSING IN ACTRIS}

Aerosol remote sensing in ACTRIS builds on the heritage of EARLINET and AERONET-Europe and applies standards developed for these observation networks over the past two decades. ACTRIS requires lidar-photometer synergy, i.e., the equipment at the Observational Platforms always consists of at least a high-power aerosol lidar and an automatic sun/sky (and lunar) photometer [4]. The distribution of aerosol remote-sensing sites over Europe as foreseen by 2025 is shown in Figure 2.

ACTRIS high-power aerosol lidars provide quantitative measurements of aerosol extinction and backscattering profiles throughout the troposphere and lower stratosphere with high spatial and temporal resolution. Spectral and polarization information (extinction-related and backscatter-related Ångström exponents and particle linear depolarization ratio) is retrieved in order to classify aerosol types and to determine natural and anthropogenic contributions to the aerosol load. ACTRIS variables also include 
information on geometrical boundaries of aerosol layers and layer-mean optical properties.

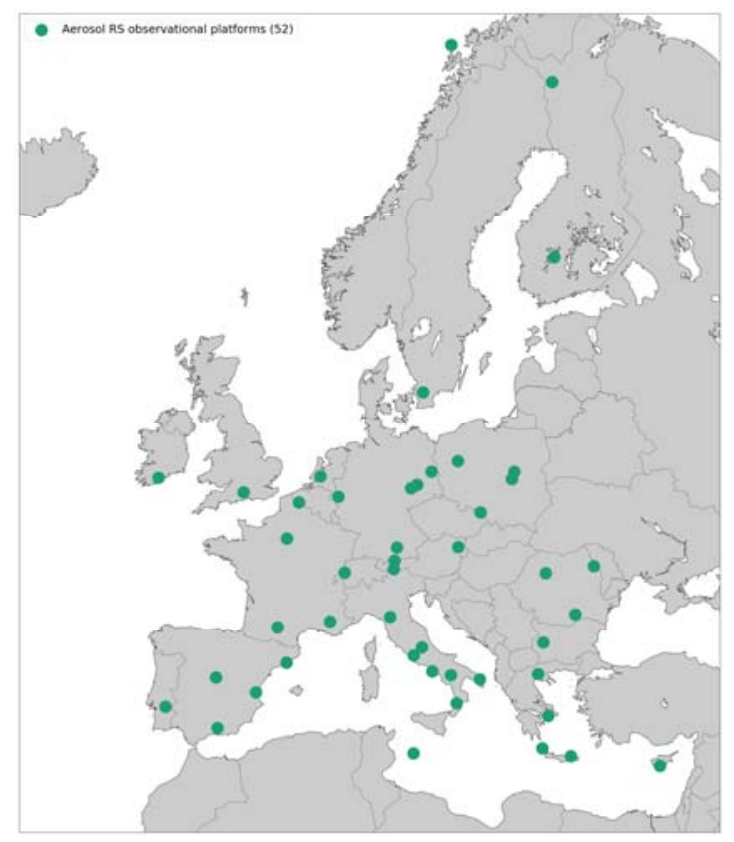

Figure 2: Distribution of ACTRIS aerosol remotesensing sites over Europe as foreseen by 2025. Several additional sites are located outside of Europe (Arctic, Tropics, Central Asia).

Automatic sun/sky/lunar photometers measure the columnar spectral aerosol optical depth and sky radiance, from which information on particle microphysical and radiative properties, e.g., particle size distribution, absorption, complex refractive index, spherical/non-spherical fraction, phase matrix, is retrieved. The synergistic use of photometer and lidar observations aims at the retrieval of higher-level variables such as fine and coarse particle mass concentration and spectral extinction, backscatter and absorption profiles.

In general, ACTRIS recommends an optimum measurement setup for its Observational Platforms and requires at least a minimum configuration in order to qualify as a National Facility and receive the respective label. The optimum setup of an ACTRIS aerosol remotesensing platform consists of a three-wavelength Raman or high-spectral-resolution (HSR) lidar with polarization discrimination capability and an automatic sun/sky/lunar photometer (optionally also polarized), both operating continuously. Instrument Level 0 data must be uploaded automatically for central processing in real real- time (RRT, data available within 3 hours after acquisition), with the aim to provide products for assimilation purposes, fast model evaluation and immediate information of the public about special events and hazardous situations.

As a minimum setup, a one-wavelength Raman or HSR lidar with polarization discrimination capability and a sun/sky photometer are required. The photometer is operated automatically, and the Level 0 data are uploaded for central processing in RRT or near real-time (NRT, data available within 3 days after acquisition). The lidar must be operated following the measurement schedule for climatological observations and shall in addition contribute to the observation of special events and satellite validation activities. Lidar data have to be uploaded in RRT or NRT in order to provide immediate public Level 1 images (quicklooks).

All higher-level variables are centrally produced, quality controlled and provided to the users through the Centre for Aerosol Remote Sensing and the ACTRIS Data Centre. Calibration and quality assurance tools for photometer and lidar instruments have to be applied following the guidelines of the Centre for Aerosol Remote Sensing.

\section{CENTRE FOR AEROSOL REMOTE SENSING (CARS)}

The main duty of the Centre for Aerosol Remote Sensing (CARS) is to enable the functionality of the National Facilities operating high-power aerosol lidars, automatic low-power aerosol lidars/ ceilometers (used for cloud remote sensing in ACTRIS) and sun/sky/lunar photometers and to assure the compliance of their methodologies and products with the requirements of full traceability, high quality and full documentation. The operation support provided by CARS to National Facilities consists of: a) procedures and tools for quality assurance and quality control of ACTRIS measurements and data; b) transfer of knowledge and training to ACTRIS operators; c) improvement of measurement methodologies. In addition, CARS will provide services for users.

CARS consists of eight Units operated by seven institutions in Romania, Germany, Italy, France and Spain, out of which three are dedicated to high-power lidars, two to low-power lidars and 
ceilometers and three to sun/sky/lunar photometers. Each Unit is specialized in providing certain services, however crucial services are covered simultaneously by at least two Units in order to minimize the risks and reduce the costs. The three Units responsible for the quality assurance of high-power lidars have been operating the Lidar Calibration Centre (LiCal) during the ACTRIS-2 project. During the ACTRIS Implementation Phase (2020-2024), their task will be to document and help in implementing, at all ACTRIS National Facilities for aerosol remote sensing, the standards and calibration techniques developed previously in the framework of EARLINET and ACTRIS, e.g., calculation of Rayleigh scattering coefficients, Rayleigh fit, trigger-delay determination, telecover test, dark-signal subtraction and polarization calibration. These tests, when used regularly and properly, are the basis for the QA/QC program of the ACTRIS high-power aerosol lidars, assuring a controlled quality of the Level 0 datasets, which are then processed with the Single Calculus Chain (SCC) in the Data Centre to produce Level 1 to Level 3 ACTRIS data. CARS support will impact on data quality mainly through avoiding signal disturbances, which may not be completely compensated by the SCC. Through training, consultancy, direct comparisons with reference systems and other specific activities, CARS will facilitate the labelling of the National Facilities.

In the following years, when ACTRIS is fully operational, CARS will work on new developments with the scope of: a) facilitating fast and correct use of the QA program by the ACTRIS operators and users on a regular basis; $b$ ) establishing new tools to quantify signal disturbances; c) producing complete error analysis of the final products, which includes and combines systematic and random errors. Also, CARS will consider future lidar developments as, e.g., the HSR lidar and measurements of the circular depolarization ratio.

\section{SUMMARY AND OUTLOOK}

ACTRIS shall establish, operate and develop a pan-European distributed research infrastructure for short-lived atmospheric constituents. Among other European observation and exploration capacities, it fosters the integration of EARLINET and AERONET-Europe in a synergistic aerosol remote sensing component, supported by the Centre for Aerosol Remote Sensing and the Data Centre. In the near future, ACTRIS shall provide effective access for a wide user community, including virtual and remote access to data and services as well as physical access to Topical Centres and National Facilities.

Currently, ACTRIS is in the Preparation Phase, which is supported by the EC-funded ACTRIS Preparatory Phase Project (PPP, 2017-2019). ACTRIS plans to enter the Implementation Phase in 2020 and to be fully operational in 2025 . The ACTRIS ERIC shall be in place in the beginning of 2021. This legal entity with statutory seat in Finland will govern the long-term sustainable operation of the research infrastructure and facilitate the interaction with the worldwide user community over the next decades.

\section{ACKNOWLEDGEMENTS}

This work has received funding by the European Commission under the Horizon 2020 - Research and Innovation Framework Programme, Grant Agreement no. 654109 (ACTRIS-2) and Grant Agreement no. 739530 (ACTRIS-PPP).

\section{REFERENCES}

[1] ACTRIS Stakeholder Handbook 2018, http://www.actris.eu/Outreach/Publications/ACTRISSt akeholderHandbook.aspx

[2] ACTRIS Data Policy, https://www.actris.eu/Portals/46/Documentation/ACTR IS PPP/Deliverables/Public/WP2_D2.3_M30.pdf

[3] ACTRIS Access and Service Policy, https://www.actris.eu/Portals/46/Documentation/ACTR IS PPP/Deliverables/Public/WP2_D2.6_M32.pdf

[4] Documentation on technical concepts and requirements for ACTRIS observational platforms, https://www.actris.eu/Portals/46/Documentation/ACTR IS\%20PPP/Deliverables/Public/WP5 D5.1_M18.pdf

[5] Documentation on technical concepts and requirements for ACTRIS exploratory platforms, https://www.actris.eu/Portals/46/Documentation/ACTR IS PPP/Deliverables/Public/WP5 D5.2 M18 .pdf

[6] Document defining the founding articles for the legal entity,

https://www.actris.eu/Portals/46/Documentation/ACTR IS PPP/Deliverables/Public/WP2_D2.4_M32.pdf 